\title{
Robust Pelvic Coordinate System Determination for Pose Changes in Multidetector-row Computed Tomography Images
}

\author{
Syoji Kobashi ${ }^{\left(1,2,{ }^{*},\right.}$, Satoshi Fujimoto ${ }^{(1}$, Takayuki Nishiyama ${ }^{(3}$, Noriyuki Kanzaki ${ }^{(3}$, Takaaki Fujishiro ${ }^{(3}$, \\ Nao Shibanuma ${ }^{(4}$, Kei Kuramoto ${ }^{(1,2}$, Masahiro Kurosaka ${ }^{(3)}$ and Yutaka Hata ${ }^{(1,2}$ \\ 1) Graduate School of Engineering, University of Hyogo \\ 2167, Shosha, Himeji 671-2280, Japan \\ 2) WPI Immunology Frontier Research Center, Osaka University, JAPAN \\ 3) Graduate School of Medicine, Kobe University, JAPAN \\ 4) Kobe Kaisei Hospital, JAPAN \\ E-mail : kobashi@eng.u-hyogo.ac.jp
}

\begin{abstract}
For developing navigation system of total hip arthroplasty (THA) and evaluating hip joint kinematics, 3-D pose position of the femur and acetabulum in the pelvic coordinate system has been quantified. The pelvic coordinate system is determined by manually indicating pelvic landmarks in multidetector-row computed tomography (MDCT) images. It includes intra- and inter-observer variability, and may result in a variability of THA operation or diagnosis. To reduce the variability of pelvic coordinate system determination, this paper proposes an automated method in MDCT images. The proposed method determines pelvic coordinate system automatically by detecting pelvic landmarks on anterior pelvic plane (APP) from MDCT images. The method calibrates pelvic pose by using silhouette images to suppress the affect of pelvic pose change. As a result of comparing with manual determination, the proposed method determined the coordinate system with a mean displacement of $2.6 \pm 1.6 \mathrm{~mm}$ and a mean angle error of $0.78 \pm 0.34 \mathrm{deg}$ on 5 THA subjects. For changes of pelvic pose position within $10 \mathrm{deg}$, standard deviation of displacement was $3.7 \mathrm{~mm}$, and of pose was $1.28 \mathrm{deg}$. We confirmed the proposed method was robust for pelvic pose changes.
\end{abstract}

Key Words : pelvic coordinate system, anterior pelvic plane, MDCT images, total hip arthroplasty

\section{Introduction}

Quantification of 3-D pose position of the femur and acetabulum is required for evaluating the hip joint kinematics or developing navigation system of total hip arthroplasty (THA) 00. By analyzing the hip joint kinematics, biomechanics of joint, mechanism of walking on two legs, the cause of joint dislocation, etc. has been studied 0 . To analyze the hip joint kinematics, 2-D/3-D image registration of 2-D X-ray images and 3-D multidetector-row computed tomography (MDCT) images has been used 00 . The methods produce 3-D pose position parameters of the femur and the pelvis in MDCT image coordinate system. Thus, to investigate the relative pose position between the femur and the acetabulum, it requires a definition of pelvic coordinate system.

Pelvic coordinate system is a coordinate system derived from subject's pelvic shape. There are mainly two definitions of the coordinate system. The representative one, and used in this study, is based on anterior pelvic plane (APP) 00. APP is a tangent plane of the pelvis, and is determined by four pelvic

Manuscript received Dec. 16, 2009; revised Jan. 10, 2010.

* Corresponding author

This work was supported in part by Ishikawa Hospital, JAPAN and Berkeley Initiative in Soft Computing (BISC) Program of University of California at Berkeley, USA. landmarks that are the right and left anterior superior iliac spines (ASIS), and the right and left pubic tubercles. The other definition of pelvic coordinate system is based on the ASIS and the posterior superior iliac spine (PSIS) 0 .

The pelvic landmarks have been determined manually in MDCT images. The manual interaction causes intra- and interuser variability. Furthermore, subjects' pose in MDCT scanner changes in every image acquisition. In such cases, it is difficult to define the same coordinate system every time. The variability of coordinate system definition affects the hip joint kinematics evaluation and functional test. Furthermore it may cause the serious problem of variability of THA operation. Therefore a robust pelvic coordinate system determination without pose change affect is required.

There are few studies for automated definition of pelvic coordinate system or for automated finding of APP. In order to find the APP, they extracts the anatomical landmarks by registering the predefined atlas data with MDCT images of evaluating subject 00 . The other method finds APP by using the mirror plane associated with the left and right ASIS 0 . Although, the conventional methods did not consider about robustness for pelvic pose changes.

This paper proposes a fully automated pelvic coordinate system in MDCT images. The method calibrates the pelvic pose by using silhouette images, and finds the pelvic landmarks and APP. Using the obtained APP and pelvic landmarks, the 


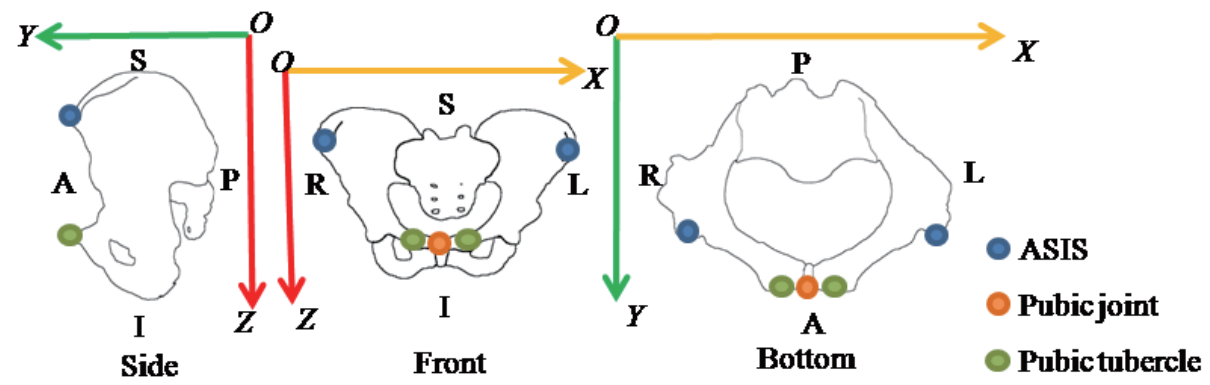

Fig. 1 Pelvic landmarks. A: Anterior, P: Posterior, R: Right, L: Left, I: Inferior, S: Superior.

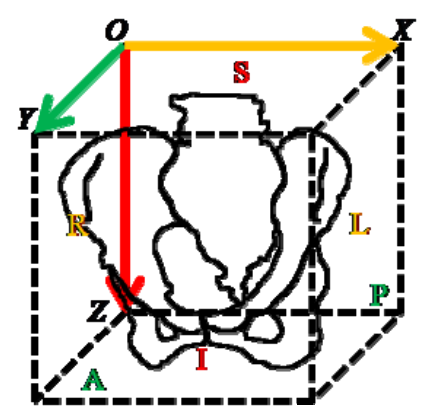

Fig. 2 MDCT coordinate system. pelvic coordinate system is defined. This method reduces variability due to pose changes and user interaction. The proposed method was applied to MDCT images of 5 THA subjects, 1 non-THA subject for qualitative evaluation, and to computer-synthesized images for quantitative evaluation.

\section{Preliminaries}

\subsection{Pelvic landmarks}

The proposed method determines the pelvic coordinate system by extracting APP. APP is a tangent plane which contacts with the anterior pelvic region. Because the contacting points are pelvic landmarks, APP can be determined by extracting the pelvic landmarks. Fig. 1 introduces location of pelvic landmarks; superior and right landmark is called the right anterior superior iliac spine (ASIS), superior and left landmark is called the left ASIS, inferior and right landmark is called the right pubic tubercle, inferior and left landmark is called the left pubic tubercle, and center of the right and the left pubic tubercles is called pubic joint.

\subsection{MDCT images}

In this study, MDCT images were acquired from an MDCT scanner (Somatom Plus, Siemens, Germany) with a matrix size of 512 by 512 pixels, a spatial resolution of about $0.75 \mathrm{~mm}$ square, number of slice of about 300 , and a slice thickness of $1.0 \mathrm{~mm}$. Subjects were scanned with a feet-first supine (FFS) pose in MDCT scanner. MDCT coordinate system is a righthanded coordinate system $(X-Y-Z)$ illustrated in Fig. 2.

In preprocessing, pelvic region is extracted by thresholding using an experimentally determined parameter, $T h_{\text {bone }}$. In addition, in case of THA patients, MDCT images are corrupted by the metal artifact (See Fig. 3). The metal artifact should be removed because it decreases the performance of finding pelvic landmarks. A voxel which satisfied formula (1) is classified into metal artifact, and is removed.

$$
\operatorname{card}\left(v \mid v \in \Delta(x), f(v) \geq T h_{\text {bone }}\right) \leq T h
$$

where $x$ is a voxel of interest, $\Delta(x)$ is a set of neighboring voxels of $x, f(v)$ is CT value of voxel $v$, and 26-neighbors are used as neighboring voxels. This formula is satisfied when the number of neighboring voxels whose $\mathrm{CT}$ value is higher than

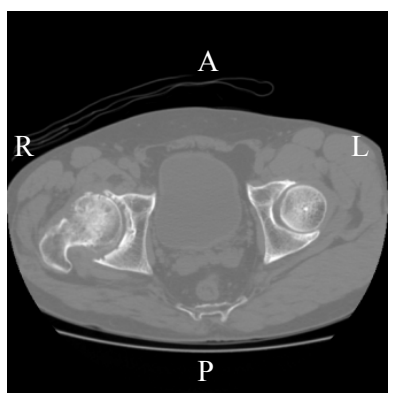

(a) Non-THA subject.

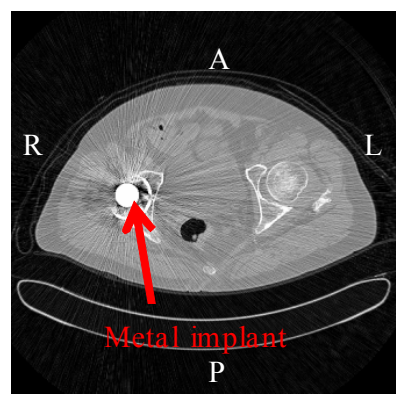

(b) THA subject.
Fig. 3 MDCT images.

$T h_{\text {bone }}$ is lower than $T h . T h$ is an experimentally determined parameter.

\section{Automated determination of pelvic coordinate system}

\subsection{Overview}

The pelvic coordinate system is determined by finding APP, the left and right ASIS, and the pubic joint in MDCT images according to literatures [9][10]. To suppress variability due to pelvic pose change, the pelvic pose is calibrated by using silhouette images. And, from the voxels belonging to pelvic region, contacting points of the pelvic region to APP can be limited to some hundred voxels using a priori knowledge. Among the limited voxels, APP is determined by using an optimization technique. Finally, the left and right ASIS and the pubic joint are extracted, and then the pelvic coordinate system is determined. In summary, the proposed method consists of the following steps.

Step 1) Calibrate pelvic pose using silhouette images.

Step 2) Limit candidates of pelvic landmarks.

Step 3) Optimize APP and extract pelvic landmarks.

Step4) Determine pelvic coordinate system.

\subsection{Calibrate pelvic pose using silhouette images}

To suppress the affect of pelvic pose change in MDCT scanner, pelvic pose is calibrated after acquiring MDCT images. The calibration is based on finding a set of rotation angles that faces the largest area of the anterior pelvis to the front $(Z-X$ 


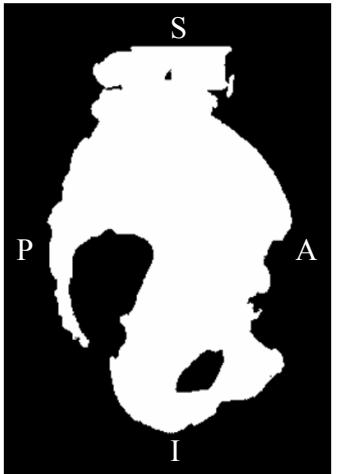

(a) Side view.

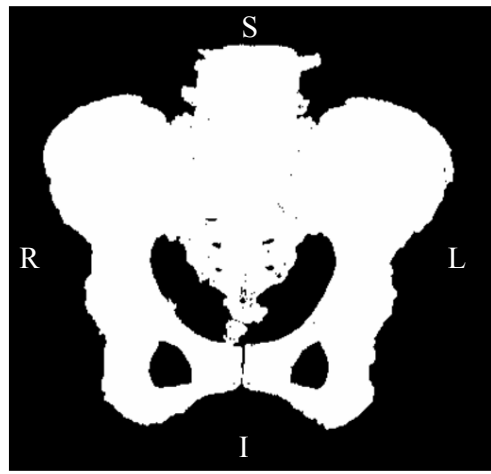

(b) Frontal view.
Fig. 4 Silhouette images.

plane) and faces the smallest area of the lateral pelvis to the side ( $Y$ - $Z$ plane) as shown in Fig. 4.

First, the pelvic region is rotated around $Y$ axis and $Z$ axis so that silhouette image of the pelvic region projected for $Y-Z$ plane becomes the smallest area. The silhouette image is synthesized by formula (2).

$$
S I_{\theta}(y, z)=\coprod_{x} I_{\theta}(x, y, z)
$$

where $\coprod$ is disjoint union of sets for $x$ and $I_{\theta}(x, y, z)$ is a value ( 0 or 1$)$ assigned to voxel at $[x, y, z]^{T}$ in the rotated image with a set of rotation angles $\theta$. $\operatorname{SI}_{\theta}(y, z)$ takes a value whether 0 or 1 , and means the value assigned at pixel $[y, z]^{T}$ in the silhouette image. The rotation angle $\theta=\left[\theta_{x}, \theta_{y}, \theta_{z}\right]^{T}$ is found by steepest descent method as follows.

$$
\begin{gathered}
\theta^{(k+1)}=\theta^{(k)}-\alpha\left[\begin{array}{c}
0 \\
\partial f\left(\theta^{(k)}\right) / \partial \theta_{y}^{(k)} \\
\partial f\left(\theta^{(k)}\right) / \partial \theta_{z}^{(k)}
\end{array}\right] \\
f\left(\theta^{(k)}\right)=\sum_{y, z} S I_{\theta^{(k)}}(y, z)
\end{gathered}
$$

where $\theta^{(k)}$ is $k$ th parameter set of rotation angles, and $\theta^{(0)}=[0,0,0]$ is the initial value.

Second, the pelvic region is rotated around $X$ axis so that silhouette image of the pelvic region projected for $Z-X$ plane becomes the largest area by steepest descent method using the following formulas.

$$
\begin{gathered}
\theta^{(k+1)}=\theta^{(k)}-\alpha\left[\begin{array}{c}
\partial g\left(\theta^{(k)}\right) / \partial \theta_{x} \\
0 \\
0
\end{array}\right] \\
g\left(\theta^{(k)}\right)=\sum_{z, x} S I_{\theta^{(k)}}(z, x)
\end{gathered}
$$

$$
S I_{\theta}(z, x)=\coprod_{y} I_{\theta}(x, y, z)
$$

\subsection{Limit candidates of pelvic landmarks}

Location of pelvic landmarks can be limited by the following anatomical (priori) knowledge; (1) the right ASIS area is located in the superior and right area; (2) the left ASIS is located in the superior and left area; (3) the right pubic tubercle is located in the inferior and right area; (4) the left pubic tubercle is located in the inferior and left area. In the limited areas, voxels with the largest $Y$-coordinate value for each pixel in $Z$ - $X$ plane is extracted as center of candidates for pelvic landmarks. When one or more pixels are given, center of them is used.

For each pelvic landmark, consider a sphere whose radius is $r$ and center is the center of candidates, and voxels in the sphere are extracted as candidates of the pelvic landmark. Candidates for the right ASIS is denoted by $V_{1}$, for the left ASIS is denoted by $V_{2}$, for the right pubic tubercle is denoted by $V_{3}$ and for the left pubic tubercle is denoted by $V_{4}$.

\subsection{Optimize APP and extract pelvic landmarks}

APP is determined from the candidate voxels by using an optimization technique. APP is a tangent plane which is determined by the right and the left ASIS, and either or both of the right or/and the left pubic tubercle because a plane is determined by three points. So, APP is determined by the following formula.

$$
\begin{aligned}
& \left(\hat{p}_{1}, \hat{p}_{2}, \hat{p}_{3}\right)=\arg \min f\left(p_{1}, p_{2}, p_{3}\right) \\
& p_{1} \in V_{1}, p_{2} \in V_{2}, p_{3} \in V_{3} \cup V_{4}
\end{aligned},
$$

where $f\left(p_{1}, p_{2}, p_{3}\right)$ is a function of counting the pelvic voxels whose location is superior from a plane determined by $p_{1}, p_{2}$ and $p_{3}$. As the result, three points that are located on the right ASIS, on the left ASIS, and on the right or left public tubercle are obtained, and the optimized plane is obtained. Finally, pelvic landmarks are extracted by finding gravity center of voxels of their candidates on the optimized plane, or the nearest voxel to the optimized plane among their candidates when no voxel is on the optimized APP.

\subsection{Determine pelvic coordinate system}

Using the obtained pelvic landmarks, the pelvic coordinate system is determined. In this study, pelvic coordinate system is defined according to literatures 00 . Pelvic coordinate system is a left-handed coordinate system $\left(X_{P C S^{-}} Y_{P C S^{-}} Z_{P C S}\right)$. The origin, $O_{P C S}$, is a pubic joint that is a midpoint of the right and the left pubic tubercles. The $X_{P C S}$ axis is parallel to a straight line connecting the left ASIS and the right ASIS. The $Y_{P C S}$ axis is directed vertically for APP. The $Z_{P C S}$ axis is an outer product of $X_{P C S}$ axis and $Y_{P C S}$ axis. Fig. 5 illustrates the pelvic coordinate system definition. 


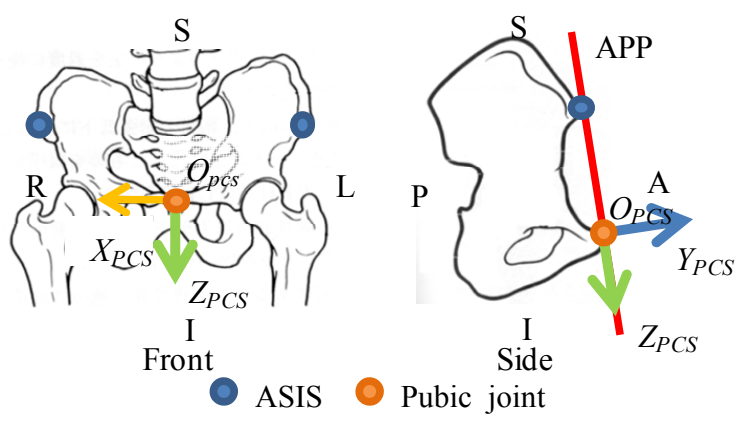

Fig. 5 Pelvic coordinate system.

\section{Experimental results}

To assess the proposed method, we have recruited five THA patients (Subject \#1 to \#5) and non-THA patient (subject \#6). All subjects gave their informed consent according to the guidelines approved by Kobe University, JAPAN. Same analysis parameters were used: $T h_{\text {bone }}$ was $210 \mathrm{HU}$; $T h$ was 9 voxels; $r$ was $10 \mathrm{~mm}$; $\alpha$ was $1.0 \times 10^{-3}$. By applying the proposed method to the acquired MDCT images, APP and the pelvic landmarks were determined. The extracted APP and landmarks for THA subject \#1 to \#5 and for non-THA subect $\# 6$ are shown in Fig. 6. For any subject, the pelvic coordinate system was defined by using the extracted landmarks according to the definition although there are differences in pelvic pose among subjects. As shown in these figures, the extracted pelvic landmarks located anterior of the pelvis and on the edges. Using the extracted landmarks, APP was determined successfully.

\subsection{Evaluation of validity}

To evaluate validity of the proposed method, the experimental results were compared with manual determination results by four well-trained orthopaedic doctors. Each doctor indicated the pelvic landmarks in multiplanar reconstruction (MPR) images. For each THA patient and for each doctor, two or more tests were performed to reduce the observer variability. Fig. 7 shows a comparison of the obtained landmarks by the proposed method with those by manual determination for subject \#1. Table 1 tabulates the displacement of origin and difference of pose. In this table, a mean of the manually given points was used as ground-truth value. On five THA subjects, the mean \pm standard deviation (SD) of origin displacement was $2.6 \pm 1.6 \mathrm{~mm}$, and of pose difference was $0.78 \pm 0.34 \mathrm{deg}$. These results show that the proposed method extracted pelvic landmarks that were similar to those given manually by the orthopaedic doctors.

\subsection{Evaluation of robustness for pelvic pose change}

To evaluate the robustness for pelvic pose changes with the proposed method, MDCT images of subject \#4 were rotated by randomly chosen angles. 200 patterns were synthesized with an angle in which root-squared angle $\varphi=\sqrt{\varphi_{x}^{2}+\varphi_{y}^{2}+\varphi_{z}^{2}}$ was

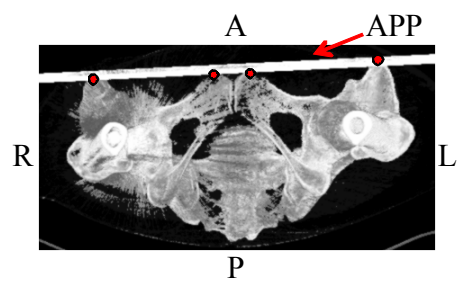

(a) Subject \#1

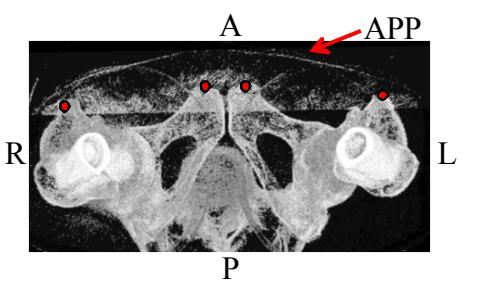

(c) Subject \#3

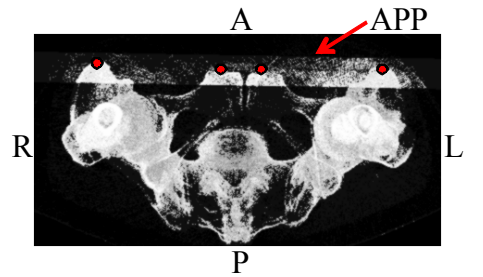

(e) Subject \#5
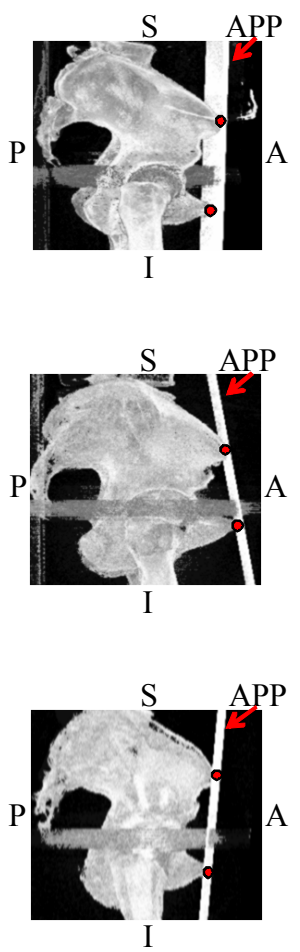

Fig. 6 Extracted pelvic landmarks and determined APP with the proposed method. Left: Inferior-superior view, Right: Left-right
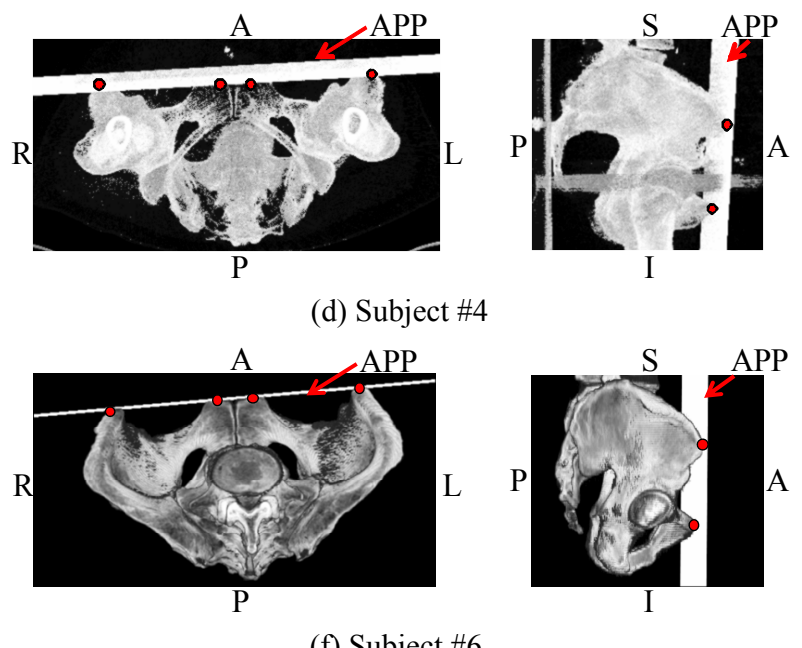

(d) Subject \#4 view, O: Pelvic landmarks, and white planes are the determined APP. 

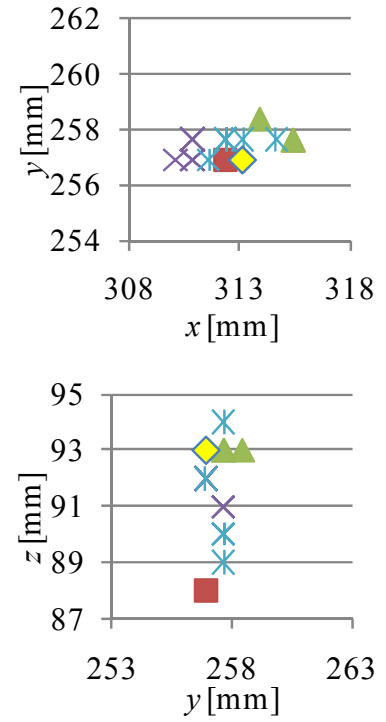

(a) Left ASIS
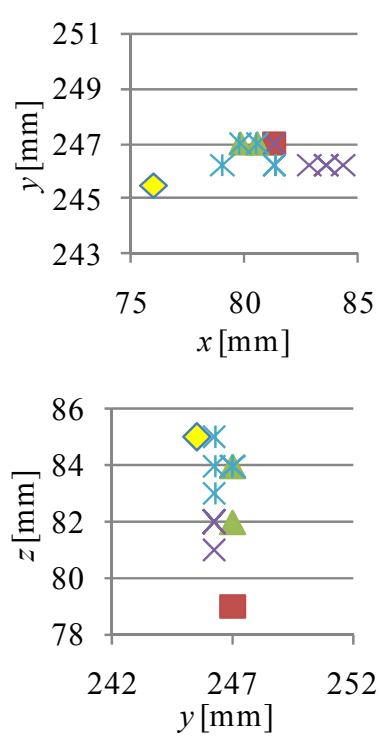

(b) Right ASIS
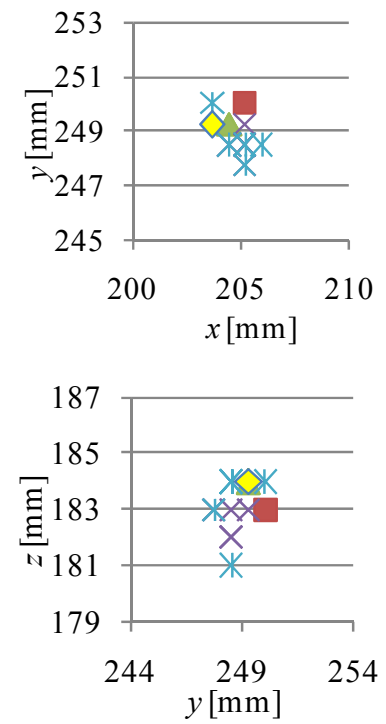

(c) Left pubic tubercle
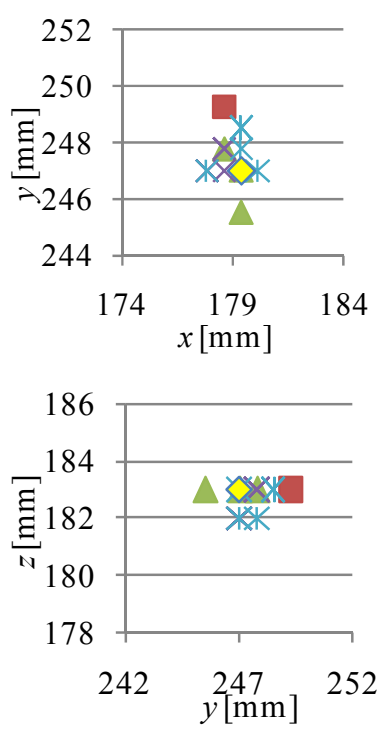

(d) Right pubic tubercle

Fig. 7 Comparison of the extracted landmarks by the proposed method with those by manual determination (Subject \#1).

$\square:$ doctor $1, \Delta$ : doctor $2, x$ : doctor 3 , : doctor $4, \diamond:$ proposed method.

less than $30 \mathrm{deg}$. The proposed method determined the pelvic coordinate system for all patterns. Fig. 8 shows distribution of SD for pelvic pose change. Even if the rotation angle $\varphi$ becomes big, the proposed method obtained a good result that $\mathrm{SD}$ is small. The results showed that the SD of origin displacement was $3.7 \mathrm{~mm}$ and pose difference was $1.28 \mathrm{deg}$. This indicates the high robustness of the proposed method for the pelvic pose change.

\section{Discussion}

Fig. 9 shows distribution of pelvic landmarks that were obtained from randomly rotated MDCT images of a THA subject (subject \#1) and of a non-THA subject (subject 6) with a similar manner described in Sec. 4.2. A mean SD for THA subject was $1.5 \mathrm{~mm}$ and a mean SD for non-THA subject was $1.0 \mathrm{~mm}$. SD of the extracted pelvic landmarks for non-THA subject was smaller than for THA subjects. The differences indicated that metal artifact effect was still remained and the pelvic region was under-segmented or over-segmented after metal artifact reduction process as shown in Fig. 10. In this study, metal artifact reduction method was based on a spatial distribution of metal artifacts. Because metal artifacts around implants are densely existed, they cannot be removed by the present method. Since few metal artifacts were not removed around the implant, false detection of pelvic landmarks was occurred. They resulted in slightly large SD for THA subjects in comparison with for non-THA subjects.

\section{Conclusion}

This paper proposed a fully-automated pelvic coordinate system determination method in MDCT images. By automating
Table 1. Displacement of origin and difference of pose in comparison with manual determination results.

\begin{tabular}{|c||c|c|}
\hline & $\begin{array}{c}\text { Origin displacement } \\
{[\mathrm{mm}]}\end{array}$ & $\begin{array}{c}\text { Pose difference } \\
{[\mathrm{deg}]}\end{array}$ \\
\hline \hline Subject \#1 & 0.69 & 0.94 \\
\hline Subject \#2 & 1.27 & 0.46 \\
\hline Subject \#3 & 1.81 & 0.55 \\
\hline Subject \#4 & 4.69 & 0.52 \\
\hline Subject \#5 & 4.09 & 1.35 \\
\hline Subject \#6 & 3.01 & 0.83 \\
\hline
\end{tabular}

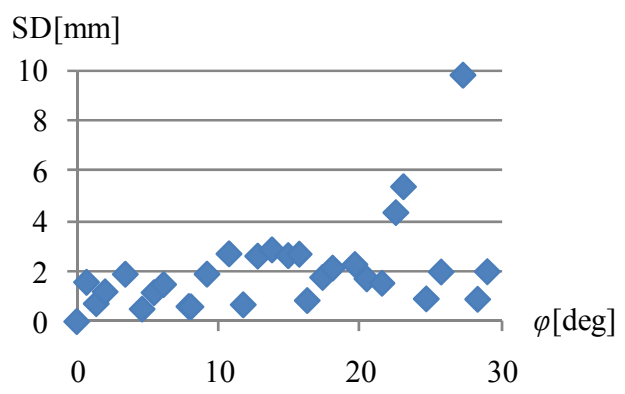

Fig. $8 \mathrm{SD}$ of pelvic landmark for pelvic pose change (right ASIS).

the pelvic coordinate system determination, the intra- and interobserver variability was removed. And, pose calibration process included in the proposed method extremely reduced the affect of pose change in MDCT scanner. By comparing manual determination method by orthopaedic doctors, the mean origin displacement was $2.6 \mathrm{~mm}$, and the mean pose difference was $0.78 \mathrm{deg}$ on five THA subjects and one non-THA subject. In addition, for pelvic pose change within $30 \mathrm{deg}$, the SD of origin displacement was $3.7 \mathrm{~mm}$, and pose was 1.28 . The differences 


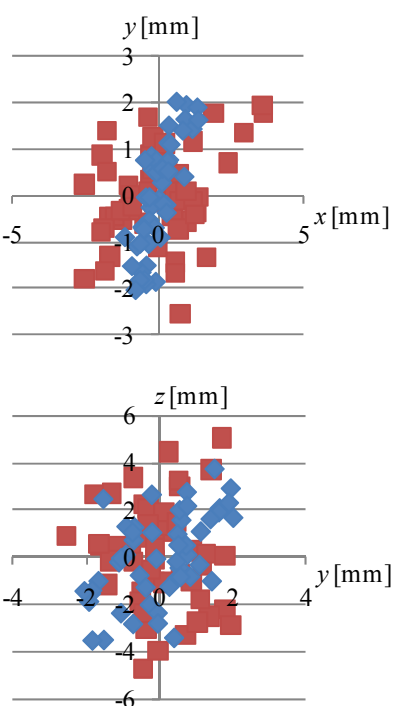

(a) Right ASIS.
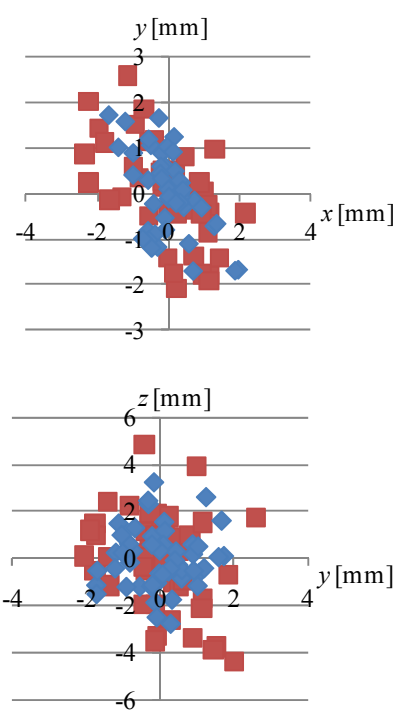

(b) Left ASIS.
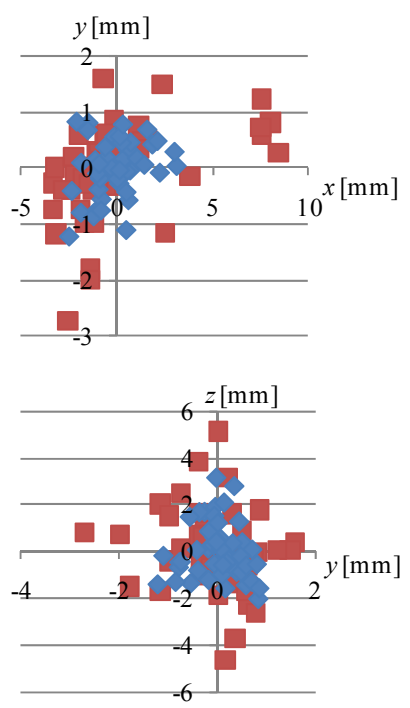

(c) Right pubic tubercle.
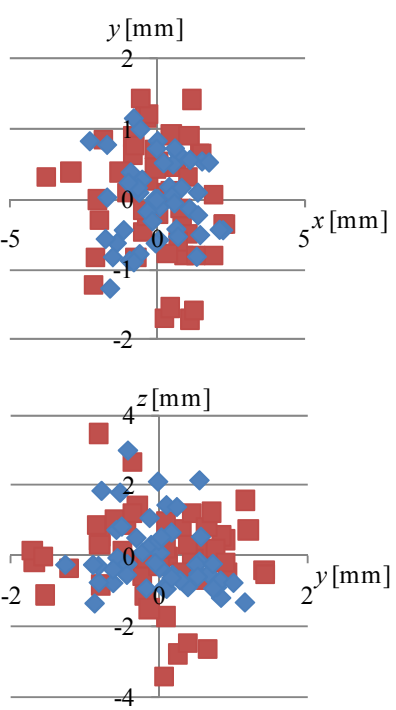

(d) Left pubic tubercle.

Fig. 9 Comparison of performance between THA subject ( $\diamond)$ and non-THA subject ( $\square$ ).

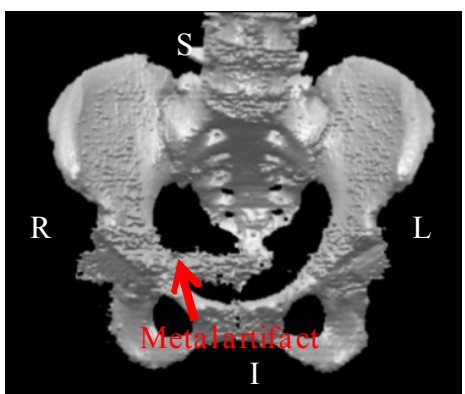

(a) Without metal artifact reduction.

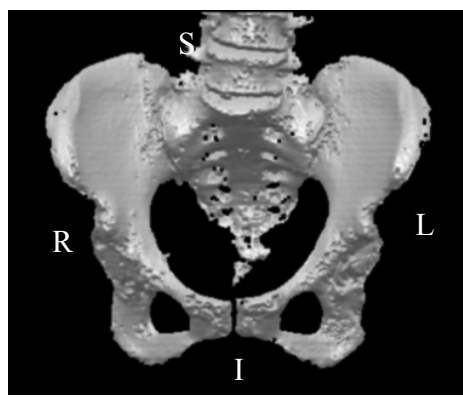

(b) With metal artifact reduction. Fig. 10 Results of metal artifact reduction.

will be caused since metallic THA implant was implanted in the subject and metal artifacts in MDCT images were still remained. Therefore, we will investigate the metal artifact reduction method to reduce the variability. And, we will validate the defined pelvic coordinate system in clinical studies. In the future, this method will be applied to estimate hip joint kinematics after THA and to define pelvic coordinate system when THA surgery.

\section{References}

[1] D. W. Murray, "The definition and measurement of acetabular orientation," The J. of Bone and Joint Surgery, vol. 75-B, no. 2, pp. 228-232, 1993.

[2] D. LaRose, L. Cassenti, B. Jaramaz, J. Moody, T. Kanade, and A. DiGioia, "Post-operative measurement of acetabular cup position using X-ray/CT Registration," Medical Image Computing and Computer-Assisted Intervention-MICCAI2000, Lecture Notes in Computer Science, vol. 1935, pp. 1104-1113, 2004.
[3] G. E. Lewinnek, J. L. Lewis, R. Tarr, C. L. Compere, and J. R. Zimmerman, "Dislocations after total hip-replacement arthroplasties," The J. of Bone Joint Surgery, vol. 60, no. 2, pp. 217-220, 1978.

[4] G. P. Penney, P. J. Edwards, J. H. Hipwell, M. Slomczykowski, I. Revie, and D. J. Hawkes, "Postoperative calculation of acetabular cup position using 2-D-3-D registration," IEEE Trans. on Biomedical Engineering, vol. 54, no. 7, pp. 13421348, 2007.

[5] M. Fukuoka, S. Kobashi, T. Nishiyama, N. Shibanuma, T. Fujishiro, S. Imawaki, M. Kurosaka, and Y. Hata, "Kinematics analysis of total hip arthroplasty with multi-modal image registration," Proc. of World Automation Congress, online, 2008.

[6] Y. S. Lee, and T. R. Yoon, "Error in acetabular socket alignment due to the thick anterior pelvic soft tissues," The $J$. of Arthroplasty, vol. 23, no. 5, pp. 699-706, 2008.

[7] A. Wolf, A. M. Digioia, A. B. Mor, and B. Jaramaz, "Cup alignment error model for total hip arthroplasty," Clinical Orthopaedic and Related Research, vol. 437, pp. 132-137, 
2005.

[8] G. Wu, S. Siegler, P. Allard, C. Kirtley, A. Leardini, D. Rosenbaum, M. Whittle, D. D. D'Lima, L. Cristofolini, H. Witte, O. Schmid, and I. Stokes; Standardization and terminology committee of the international society of biomechanics, "ISB recommendation on definitions of joint coordinate system of various joints for the reporting of human joint motion-part I: ankle, hip, and spine," J. of Biomechanics, vol. 35, no. 4, pp. 543-548, 2002.

[9] P. Foroughi, D. Song, G. Chintalapani, R. H. Taylor, and G. Fichtinger, "Localization of pelvic anatomical coordinate system using US/Atlas Registration for total hip replacement," Medical Image Computing and Computer-Assisted Intervention-MICCAI2008, Lecture Notes of Computer Science, vol. 5242, pp. 871-879, 2008.

[10] J. Ehrhardt, H. Handels, B. Strathmann, T. Malina, W. Plötz, and S. J. Pöppl, "Atlas-based recognition of anatomical structures and landmarks to support the virtual threedimensional planning of hip operations," Medical Image Computing and Computer-Assisted Intervention-MICCAI2003, Lecture Notes of Computer Science, vol. 2878, pp. 17-24, 2003.

[11] L. Fieten, J. Eschweiler, S. Heger, K. Kabir, S. Gravius, M. de la Fuente, and K. Radermacher, "Surface-based determination of the pelvic coordinate system," Medical Imaging 2009, Proceedings of the SPIE, vol. 7261, pp. 726138.1-726138.10, 2009.

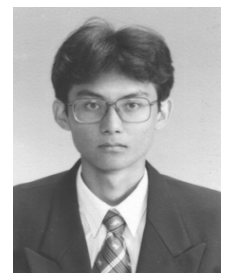

Syoji Kobashi received the B.Eng. degree in electronics in 1995, the M.Eng. degree in 1977, and the D.Eng. degree in 2000, from Himeji Institute of Technology, Hyogo, Japan. He is currently an associate professor with Graduate School of Engineering, University of Hyogo, and Associate Guest Professor, WPI Immunology Frontier Research Center, Osaka University. His research interests include soft computing, medical image processing and human brain function. He is a member of the IEICE, Biomedical Fuzzy Systems Association, Japan Society for Fuzzy Theory and Systems, and the IEEE. He received the Joseph F. Engelberger Best Paper Award from the 2nd World Automation Congress in 2000, JpCOMPEmbs03 from IEEE EMBS Japan Chapter in 2003, and Franklin V. Taylor Memorial Award from 2009 IEEE SMC annual conference. And, he is an associate editor of International Journal of Intelligent Computing in Medical Sciences and Image Processing, and of Autosoft journal.

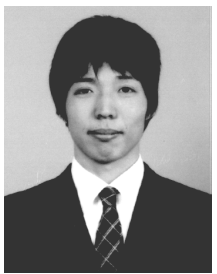

Satoshi Fujimoto received the B.Eng. degree in 2009 from University of Hyogo, Hyogo, Japan. He is currently an graduate student of University of Hyogo. His research interests include medical image processing for orthopaedics. $\mathrm{He}$ is a member of the IEEE. He received the Best Paper Award from 10th International Symposium on advanced Intelligent Systems in 2009.

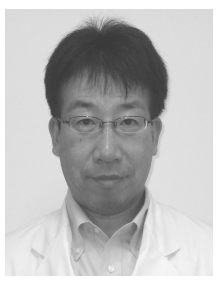

Takayuki Nishiyama is currently an associate professor with Section of Advanced Musculo-Skeletal Research, Department of Orthopaedic Surgery, Kobe University Graduate School of Medicine.

His main work is "Cis-Acting Intronic Elements that Regulate Cartilage-Specific Alternative Splicing of the Type II Collagen (Col2) Pre-mRNA Lie at or Near Splice Site Junction Sequences Flanking Exon 2 of the Gene." J Bone Miner Res. 18(9) 2003. He is a member of Orthopaedic Research Society, Japanese Orthopaedic Association, Japanese Hip Society, The Japanese Society for Surgery of the Foot (JSSF), and Japan College of Rheumatology.

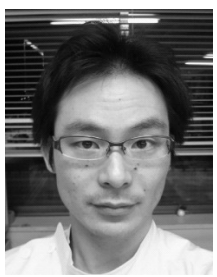

Noriyuki Kanzaki is with Department of Orthopaedic Surgery, Kobe University Graduate School of Medicine. His main work is "Involvement of the nectin-afadin complex in platelet- derived growth factorinduced cell survival," J Cell Sci. 2008 Jun 15;121(Pt 12):2008-17, Epub 2008 May 27. $\mathrm{He}$ is a member of Japanese Orthopaedic Association, Japanese Hip Society, and The Japanese Society for Surgery of the Foot (JSSF).

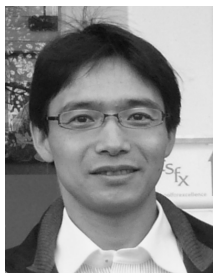

Takaaki Fujishiro is currently an assistant professor, Section of Advanced MusculoSkeletal Research, Department of Orthopaedic Surgery, Kobe University Graduate School of Medicine. His main work is "Histologic Analysis of Allograft MixedWith Hydroxyapatite-Tricalcium Phosphate Used in Revision Femoral Impaction Bone Grafting." Orthopedics 31:277, 2008. He is a member of Japanese Orthopaedic Association, Japanese Hip Society, and The Japanese Society for Surgery of the Foot (JSSF). 


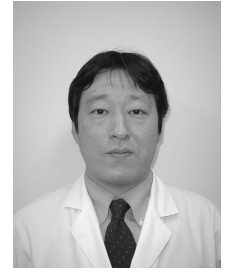

Nao Shibanuma is currently with Kobe Kaissei Hospital. His main work is "Sensitivity of femoral orientation estimates to condylar surface and MR image plane location." Journal of Magnetic Resonance Imaging, Vol. 20, pp. 300-305, 2004. He is a member of Orthopaedic Research Society, Japanese Orthopaedic Association, Japan College of Rheumatology, Japan Arthroscopy Association, Japanese Society of Reconstruction Arthroplasty, Japanese Association of Rehabilitation Medicine, and Central Japan Association of Orthopaedic Surgery \& Traumatology.

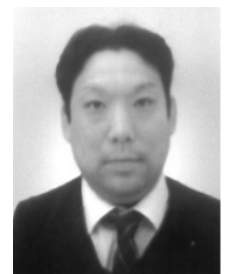

Kei Kuramoto received his B.E. degree (Education) in 1999 from Nara University of Education, his M.E. degree in 2001 from Nara Institute of Science and Technology and his D.E. degree in 2005 from Kyoto University, Japan. He is currently an associate professor of the Division of Computer Engineering, University of Hyogo. His research interests include high performance computing for medical informatics, nano chemistry and multi-physics simulation. He is a member of IEEE.

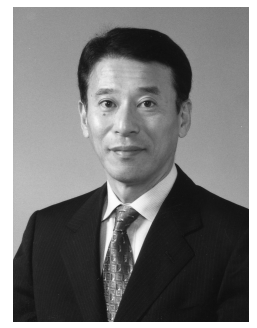

Masahiro Kurosaka is currently an professor with Department of Orthopaedic Surgery, Kobe University Graduate School of Medicine. His main work is "Maximizing flexion after total knee arthroplasty: the need and the pitfalls." Journal of Arthroplasty, Vol.17, pp. 59-62, 2002. He is a member of American Orthopaedic Society for Sports Medicine (Associated), Orthopaedic Research Society (American), European Society of Sports Traumatology, Knee Surgery and Arthroscopy (ESSKA), International Society of Arthroscopy, Knee Surgery and Orthopaedic Sports Medicine(Member at large 1999-2003) and Arthroscopy Association of North America(AANA)(Honorary 2004-).

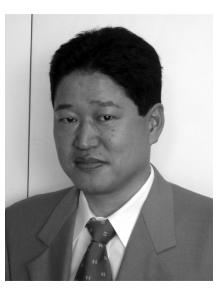

Yutaka Hata received his B.E. degree (Electronics) in 1984, his M.E. degree in 1986 and his D.E. degree in 1989 from Himeji Institute of Technology, Hyogo, Japan. He is currently a professor of the Division of Computer Engineering, University of Hyogo. He was a visiting scholar from April 1995 to March 1996 in the Computer Science Division, University of California at Berkeley. He is the chairman of BISC SIG in Medical Imaging, University of California at Berkeley. His research interests include medical informatics and soft computing. He received the Franklin V. Taylor Memorial Award for the Best Paper and Oral Presentation in 2009 IEEE International Conference on Systems, Man, Cybernetics (USA), Life-time achievement award from World Automated Congress and Automation and Soft Computing Journal, USA (2008) among others. He is a member of the Japan Society for Fuzzy Theory and Systems, Japan Society of Medical Electronics and Biological Engineering, Biomedical Fuzzy Systems Association, the Institute of Image Information and Television Engineering, The Institite of Electronics, Information and Communication Engineer in Japan, and IEEE Fellow. 